Temperature and strain-independent curvature sensor based on a singlemode/multimode fiber optic structure

This article has been downloaded from IOPscience. Please scroll down to see the full text article.

2011 Meas. Sci. Technol. 22085201

(http://iopscience.iop.org/0957-0233/22/8/085201)

View the table of contents for this issue, or go to the journal homepage for more

Download details:

IP Address: 193.136.24.86

The article was downloaded on 28/06/2011 at 20:21

Please note that terms and conditions apply. 


\title{
Temperature and strain-independent curvature sensor based on a singlemode/multimode fiber optic structure
}

\author{
Susana Silva ${ }^{1,2}$, O Frazão ${ }^{1}$, J Viegas $^{1,2}$, L A Ferreira ${ }^{1}$, F M Araújo ${ }^{1}$, \\ F Xavier Malcata ${ }^{3,4}$ and J L Santos ${ }^{1,2}$ \\ ${ }^{1}$ INESC Porto, Rua do Campo Alegre 687, 4169-007 Porto, Portugal \\ ${ }^{2}$ Departamento de Física e Astronomia da Faculdade de Ciências da Universidade do Porto, \\ Rua do Campo Alegre 687, 4169-007 Porto, Portugal \\ ${ }^{3}$ ISMAI-Instituto Superior da Maia, Avenida Carlos Oliveira Campos, 4475-690 Avioso S. Pedro, \\ Portugal \\ ${ }^{4}$ Instituto de Tecnologia Química e Biológica, Universidade Nova de Lisboa, Avenida da República, \\ 2780-157 Oeiras, Portugal \\ E-mail: jlsantos@inescporto.pt
}

Received 13 November 2010, in final form 10 May 2011

Published 28 June 2011

Online at stacks.iop.org/MST/22/085201

\begin{abstract}
This work describes a fiber optic sensing structure that is sensitive to curvature, and features a low temperature- and strain cross-sensitivity. It is based on multimode interference, and relies on a singlemode-step index multimode-singlemode fiber structure. It was observed that the transmitted optical power in such a layout is highly sensitive to the wavelength of operation, and to the length of the multimode fiber. The optical spectrum exhibits two dominant loss bands, at wavelengths that have similar responses both to temperature and strain, but different responses to curvature. Based on this result, an interrogation approach is proposed that permits substantial sensitivity to curvature $(8.7 \pm 0.1 \mathrm{~nm} \mathrm{~m})$ and residual sensitivities to temperature and strain $\left(0.3 \pm 0.1 \mathrm{pm}^{\circ} \mathrm{C}^{-1}\right.$ and $(-0.06 \pm 0.01) \times 10^{-6} \mathrm{~m} \mathrm{~m}^{-1}$, respectively). The beam-propagation method was employed for modeling the propagation of light along the optical fiber sensing device proposed.
\end{abstract}

Keywords: curvature, fiber optic sensors, multimode interference, refractive index, singlemode-multimode-singlemode structures, strain, temperature

(Some figures in this article are in colour only in the electronic version)

\section{Introduction}

The multimode interference (MMI) in a planar waveguide has been comprehensively investigated, and the self-imaging of the input light field is well known and has been widely employed in attempts to develop beam splitters, combiners and multiplexers for optical communications [1-3].

In the past few years, the MMI in optical fiber structures has been studied toward development of new optical devices [4-8]. These fiber optic devices are based on a singlemode- multimode-singlemode (SMS) fiber structure, and possess unique spectral characteristics that render them suitable for optical communications and sensing [9, 10]. Mohammed et al [4] developed an SMS bandpass filter based on MMI, and modeled light propagation within the SMS fiber structure-thus showing that it has a low insertion loss, with adequate bandwidth and isolation for coarse wavelengthdivision multiplexing. Wang et al [5] employed, in turn, a numerical beam propagation method (BMP); via scanning the length of the multimode fiber (MMF) section, these 


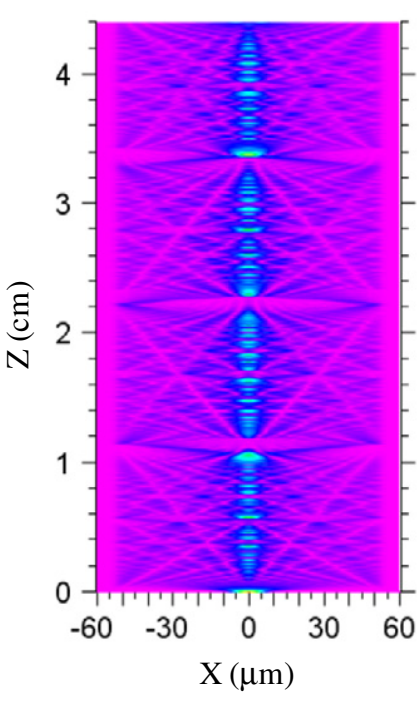

(a)

Computed Transverse Field Profile at $Z_{1}=40 \mathrm{~mm}$

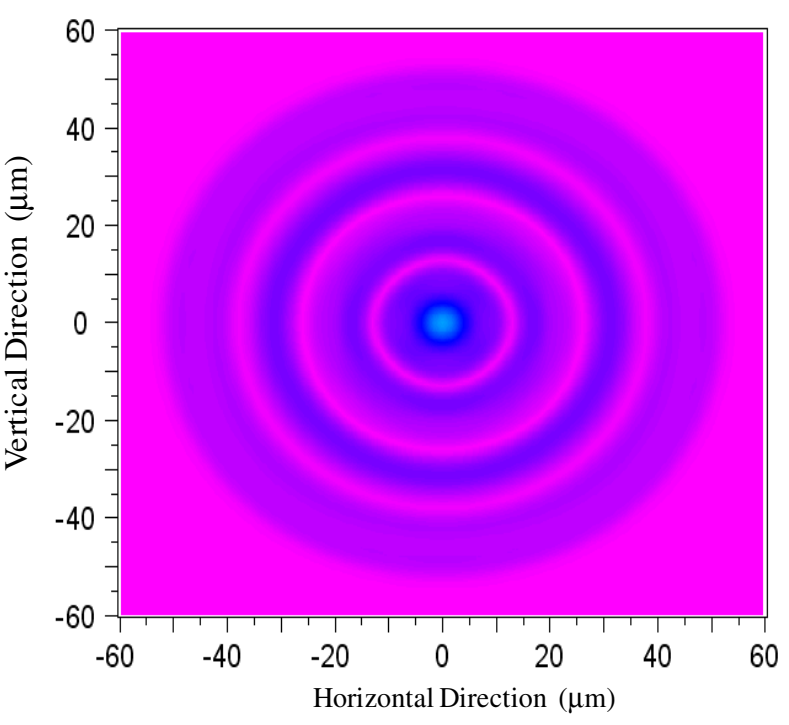

(c)

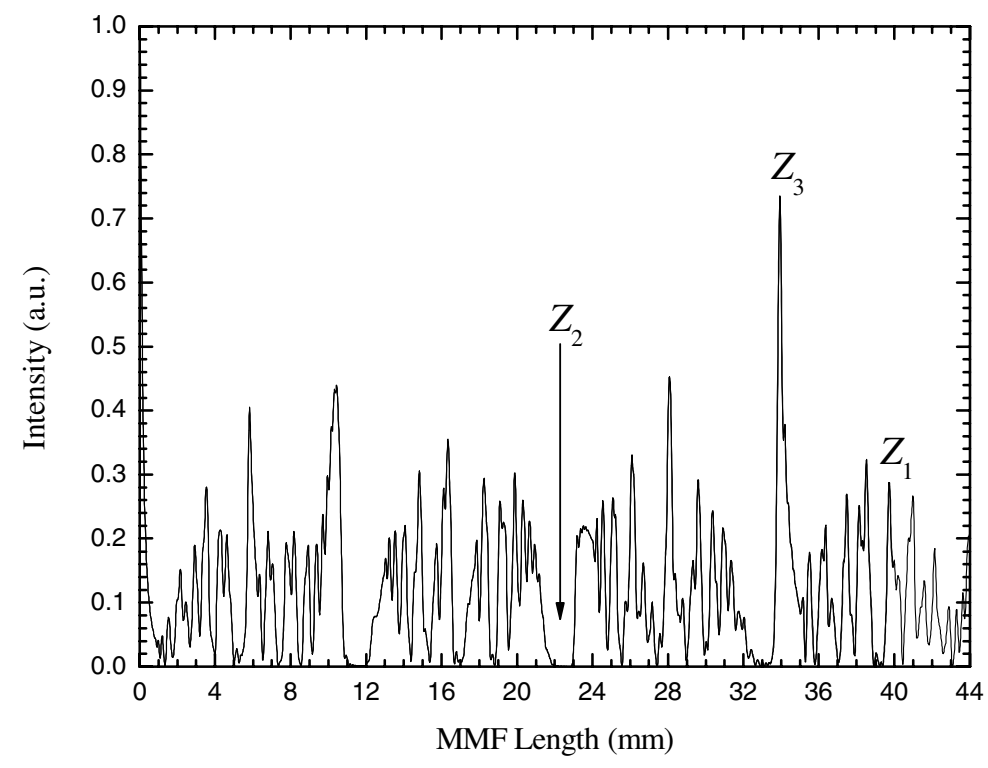

(b)
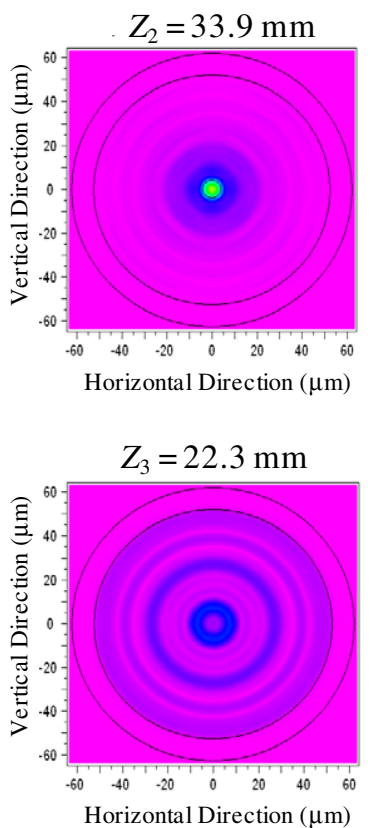

Figure 1. (a) Intensity distribution of electric field on the $x z$ plane; $(b)$ coupling overlap integral between the calculated field at a given MMF length and the input field for the simulation (SMF-28 fundamental mode); (c) mode profile of light coupled into the output SMF for an MMF length of $Z_{1}=40 \mathrm{~mm}$; inset, mode profile of light at $Z_{2}=33.9$ and $Z_{3}=22.3$ mm, corresponding to maximum coupling and no coupling of light to the output SMF, respectively.

authors found a suitable value $(\sim 9400 \mu \mathrm{m})$ for such specific applications as a refractometric sensor with an optimum refractive index measurement range of 1.38-1.45, and also an edge filter [6] with an operational wavelength range larger than $50 \mathrm{~nm}$. Furthermore, Donlagic et al [7] presented an SMS microbend sensing configuration, in which an MMF was used with a gradient index profile that provided no MMI, while the light was propagating along the fiber structure; when the proper splicing method was used, losses as low as $1.5 \mathrm{~dB}$ were indeed achieved. Furthermore, Zhang et al [8] studied a sensing scheme to measure temperature and strain simultaneously, by multiplexing a section of an MMF and a Fizeau etalon interferometer; measurement resolutions of $2.2{ }^{\circ} \mathrm{C}$ and $0.6 \mu \mathrm{m}$ were accordingly attained. Frazão et al [9] meanwhile produced a Mach-Zehnder curvature sensor based on a SMS fiber layout, where the MMI phenomenon was combined with the operation of a long-period grating; they experimentally demonstrated that, for an optimum $32 \mathrm{~mm}$ of MMF length, the SMS fiber sensor exhibited a maximum curvature sensitivity of $612.4^{\circ} \mathrm{m}$. More recently, a displacement sensor based on a bent SMS structure was reported by Wu et al [10]; such a simple scheme was able to overcome the limitations 


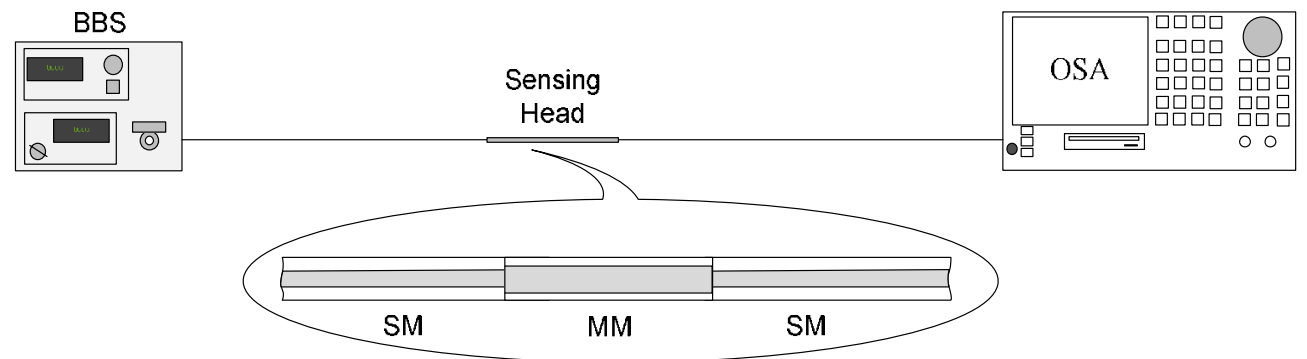

(a)

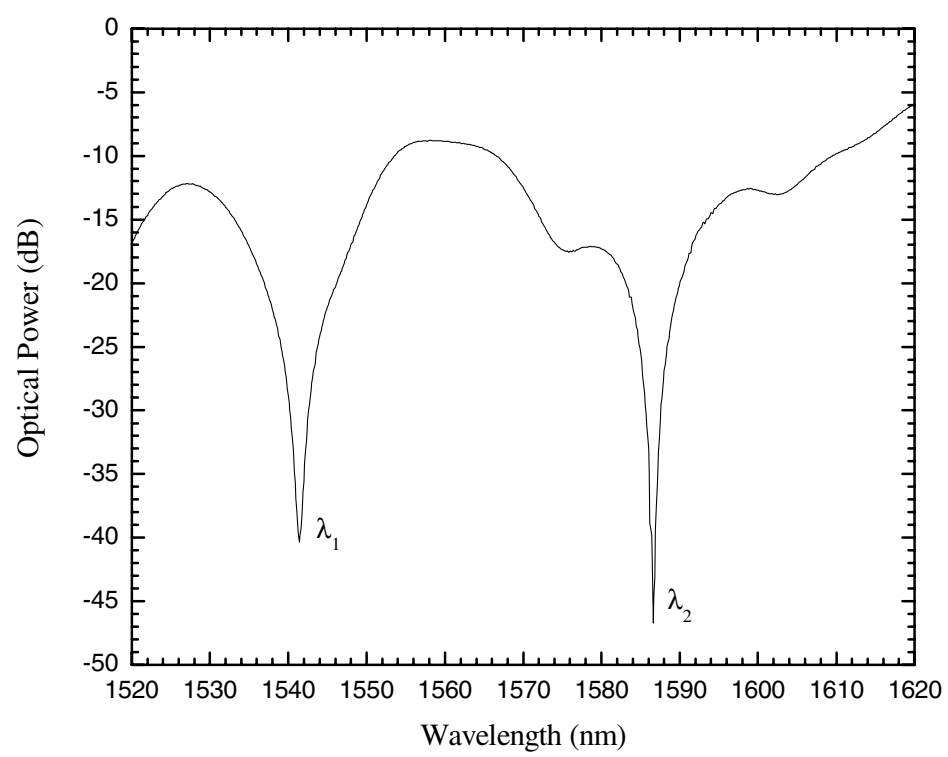

(b)

Figure 2. (a) Experimental setup with a sensing head detail, and (b) optical spectrum of the SMS fiber structure.

on displacement range imposed by using a straight SMS structure, so a sensitivity of $35.5 \mathrm{pm} \mu \mathrm{m}^{-1}$ could be attained for measurements in the 0 to $280 \mu \mathrm{m}$ range.

In this work, the experimental results of a temperatureand strain-independent curvature sensor, based on MMI, are presented. The proposed sensing device is based on a SMS fiber structure, interrogated in transmission. The optical spectrum exhibits two resonant wavelength loss peaks, with similar sensitivities to temperature and strain, but different sensitivities to curvature. The BPM was employed in modeling light propagation along the SMS fiber structure.

\section{Modeling of light propagation in the SMS structure}

Both the spectral characteristics and theoretical analysis of the SMS fiber structure have been reported elsewhere in the literature [11, 12]. Here, we develop this study further by presenting a 3D simulation based on the BPM [13], to investigate the beam behavior in the MMF section of an SMS fiber structure in transmission (this structure is shown in the sensing head detail given in figure $2(a)$ ).

Light from a singlemode fiber, SMF-28, was injected in a step-index profile MMF with the following characteristics: numerical aperture of 0.22 , and core and cladding diameters of 105 and $125 \mu \mathrm{m}$, respectively. For the operational center wavelength of $1550 \mathrm{~nm}$, the refractive indices of the core and cladding were 1.4446 and 1.4277, respectively. A singlemode fiber (SMF-28) was also used for the output of the MMF section. The SMFs and MMFs were assumed to be aligned along the same axis, and had a circular fiber cross-section. The modeling of light propagation was done for a total MMF section of $44 \mathrm{~mm}$.

The intensity distribution of the electric field at the simulated MMF length of $44 \mathrm{~mm}$ is plotted in figure 1(a) for an $x z$-cut, where $x$ is a radial coordinate and $z$ is the axial one (the input SMF is not plotted for clarity). When the light field from the SMF enters the MMF, high-order modes are excited, which create interference between different modes. Although the field profile changes along the MMF section, it remains symmetrically distributed along the direction of propagation (see figure 1(a)). Hence, different propagation distances correspond to different field profiles at the crosssection. As a result, coupling efficiency between the calculated field at the simulated MMF section and the input field given by the fundamental mode of the SMF (i.e. the transmission of the SMS fiber structure) will depend on the MMF length, as illustrated in figure $1(b)$. The mode profile of light coupled into the output SMF for an MMF length of $Z_{1}=40 \mathrm{~mm}$ (i.e. the one used in the experiment) is depicted in figure $1(c)$. 
For comparison, the inset of figure $1(c)$ shows the mode profile at $Z_{2}=33.9 \mathrm{~mm}$ and $Z_{3}=22.3 \mathrm{~mm}$ (see figure $1(b)$ ), corresponding to maximum coupling and no coupling of light to the output SMF, respectively.

\section{Experimental results}

The principle underlying the sensing-head concept is based on realization that when the light field propagating along the input SMF enters the MMF section, high-order modes of MMF are excited, so interference between different modes occurs while the beam propagates along the remainder of the MMF section. The light is coupled into the output SMF-and the coupling efficiency, for a given length $L$ of the MMF section, is wavelength-dependent. In fact, since higher order modes of the MMF are excited, the optical power coupled out to the SMF will depend on the amplitudes and relative phases of the various modes of the MMF at its output end. The transmitted optical power varies periodically with wavelength, with a period $\Delta \lambda_{2 \pi}$ given by [9]:

$$
\Delta \lambda_{2 \pi}=\frac{\pi^{2} a^{2} n_{\mathrm{co}}^{3}}{N A^{2} L}
$$

where $a$ is the MMF core radius, $n_{\text {co }}$ and $N A$ are the MMI core refractive index and numerical aperture, respectively, and $L$ is the MMF length. Therefore, this spectral periodicity shows relatively strong dependences on the variation of the MMF core radius, numerical aperture and core refractive index.

To demonstrate the proposed configuration, the sensinghead structure shown in figure $2(a)$ was built. It consists of a $40 \mathrm{~mm}$ long MMF (with a numerical aperture of 0.22 , and core and cladding diameters of 105 and $125 \mu \mathrm{m}$, respectively), spliced between two SMF-28 singlemode fibers and interrogated in transmission. In this simple approach, a broadband source in the $1550 \mathrm{~nm}$ spectral range was used, with a bandwidth of $100 \mathrm{~nm}$, coupled with an optical spectrum analyzer as interrogation unit. The optical spectrum of the light transmitted by the SMS fiber structure is depicted in figure $2(b)$.

In the said configuration, it can be observed that the optical power transmitted presents two wavelength loss bands (centered at $\lambda_{1}$ and $\lambda_{2}$ ) - which, in principle, will be sensitive to different physical parameters, namely curvature, temperature and strain. As predicted by (1), the spacing between the various resonances is inversely proportional to the MMF length - and, in this case, $\Delta \lambda_{2 \pi}$ is estimated as about $42 \mathrm{~nm}$, which is in the vicinity of the measured value (about $45 \mathrm{~nm})$.

The behavior of this structure as a curvature sensor was duly characterized. The fiber was fixed at two points, at a distance of $600 \mathrm{~mm}$ from each other; one of these points is a translation stage that allows the fiber to bend. The bending displacement, $d$, was applied to the SMS via sequential $100 \mu \mathrm{m}$ displacements. Hence, the curvature $(1 / R)$ of the fiber structure changed by $2 d /\left(d^{2}+L^{2}\right)$, where $L$ is half the distance between the two fixed points. The spectral positions of the resonant loss bands have different and linear responses to curvature variations, as shown in figure $3(a)$ with a sensitivity of $(7.3 \pm 0.1) \mathrm{nm} \mathrm{m}$ and $(-1.4 \pm 0.1) \mathrm{nm} \mathrm{m}$,

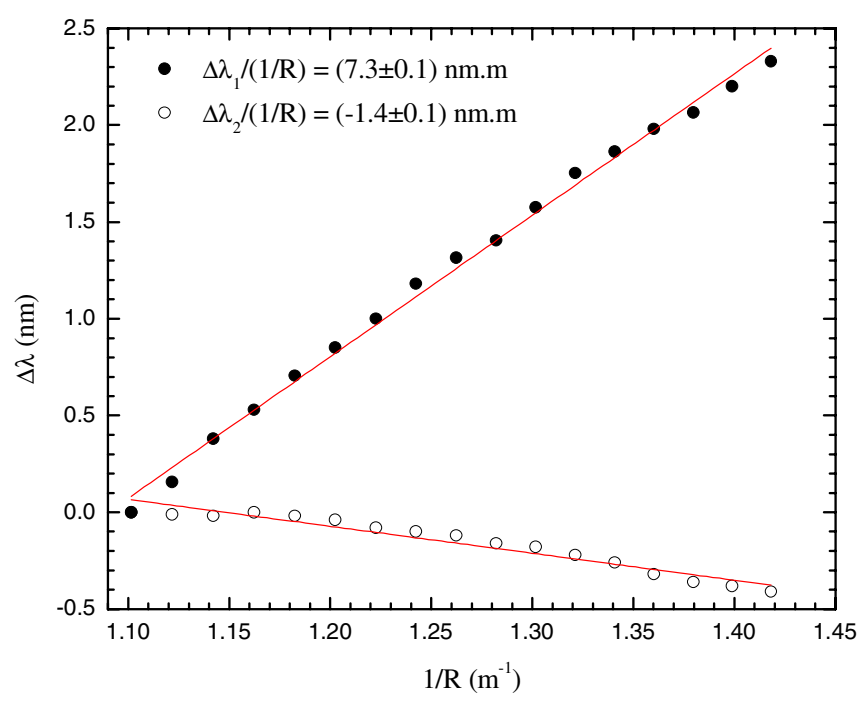

(a)

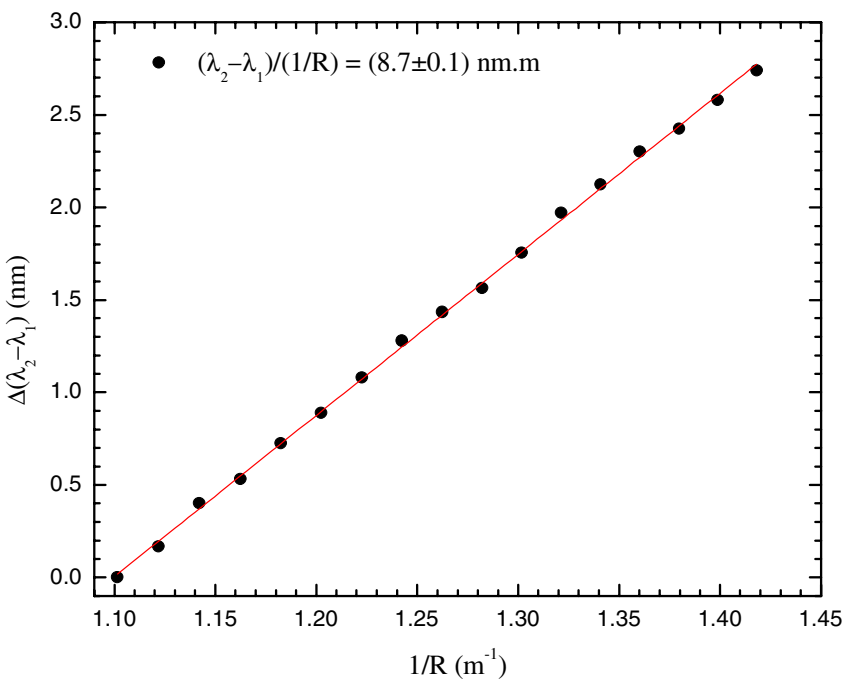

(b)

Figure 3. Curvature response for $(a)$ the two loss bands centered at $\lambda_{1}$ and $\lambda_{2}$; and $(b)$ the measurement parameter, $\Delta\left(\lambda_{2}-\lambda_{1}\right)$.

for the bands centered at $\lambda_{1}$ and $\lambda_{2}$, respectively. If the field profile were symmetrically distributed along the direction of propagation of a straight SMS structure, one should notice symmetry breaking upon application of curvature. The result would be that interference between higher-order modes is more affected by bending of the MMF section, while lower-order modes are focused within a smaller area near the fiber core and are consequently less affected by the bending disturbance. Therefore, distinct MMF modes are excited by the light exiting the input SMF, thus creating different curvature sensitivities for the resonant loss bands centered at $\lambda_{1}$ and $\lambda_{2}$. Irrespective of its origin, however, such a result indicates that the wavelength difference between these two resonances, $\Delta\left(\lambda_{2}-\lambda_{1}\right)$, can be an effective curvature measurement parameter, providing a readout sensitivity of $(8.7 \pm 0.1) \mathrm{nm} \mathrm{m}$ as shown in figure $3(b)$.

The response of the sensing head to temperature variations was also characterized, as shown in figure 4 . The structure was 


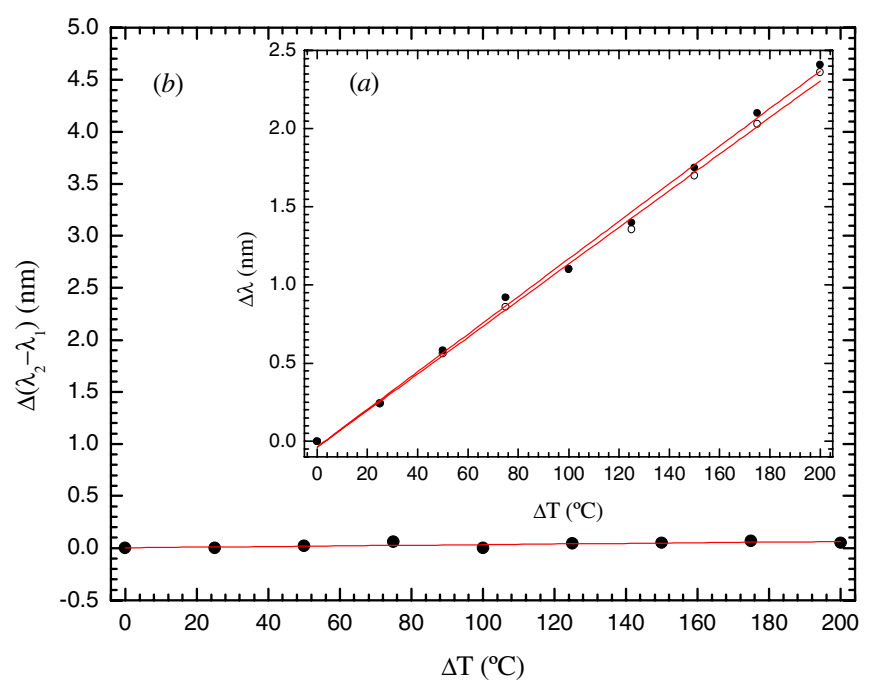

Figure 4. Temperature response for $(a)$ the two loss bands centered at $\lambda_{1}$ and $\lambda_{2}$, and $(b)$ the measurement parameter, $\Delta\left(\lambda_{2}-\lambda_{1}\right)$.

placed in a tube furnace, and submitted to increasing values of temperature in the range $0-200{ }^{\circ} \mathrm{C}$, with $25^{\circ} \mathrm{C}$ steps.

The results depicted in figure $4(a)$ indicate that the loss bands centered at $\lambda_{1}$ and $\lambda_{2}$ exhibit linear responses to temperature variations, characterized by similar sensitivities: $(11.7 \pm 0.2) \mathrm{pm}^{\circ} \mathrm{C}^{-1}$ and $(12.0 \pm 0.2) \mathrm{pm}^{\circ} \mathrm{C}^{-1}$ for $\lambda_{1}$ and $\lambda_{2}$, respectively. This was somewhat expected, since the temperature variation does not change the singlemodemultimode launching conditions; and the MMF length variation (due to thermal expansion) and MMF core refractive index variation (due to silica thermo-optic effect) only change the optical path length, with the associated wavelength scaling for the resonances. The interesting feature here is that the measurement parameter (i.e. the difference between the spectral shifts of the resonance bands, $\left.\Delta\left(\lambda_{2}-\lambda_{1}\right)\right)$ shows residual temperature sensitivity, about $(0.3 \pm 0.1) \mathrm{pm}{ }^{\circ} \mathrm{C}^{-1}$ (figure $4(b)$ ), thus substantially eliminating the problem of temperature cross-sensitivity.

The sensing structure was also characterized in terms of strain. The input and output fibers were accordingly fixed at two points that were $300 \mathrm{~mm}$ apart, and submitted to specific strain values by using a translation stage (via sequential $20 \mu \mathrm{m}$ displacements). The results are made available in figure 5(a).

Once again, the resonances have linear responses to strain variations, with similar sensitivities: $(-1.70 \pm 0.01)$ $\times 10^{-6} \mathrm{~m} \mathrm{~m}^{-1}$ and $(1.76 \pm 0.01) \times 10^{-6} \mathrm{~m} \mathrm{~m}^{-1}$, for $\lambda_{1}$ and $\lambda_{2}$, respectively; the rationale here is somewhat similar to that considered for the case of temperature. When the dependence on strain of the measurement parameter $\Delta\left(\lambda_{2}-\lambda_{1}\right)$ was evaluated, it turned out that the strain sensitivity was close to zero, about $(-0.06 \pm 0.01) \times 10^{-6} \mathrm{~m} \mathrm{~m}^{-1}$ as shown in figure $5(b)$ - so the proposed sensing configuration is also immune to the effect of strain cross-sensitivity.

Usually, optical fiber-based curvature sensors have crosssensitivity to temperature and/or strain; and several alternative sensing schemes have been proposed to overcome this limitation. A sampled chirped fiber Bragg grating (CFBG),

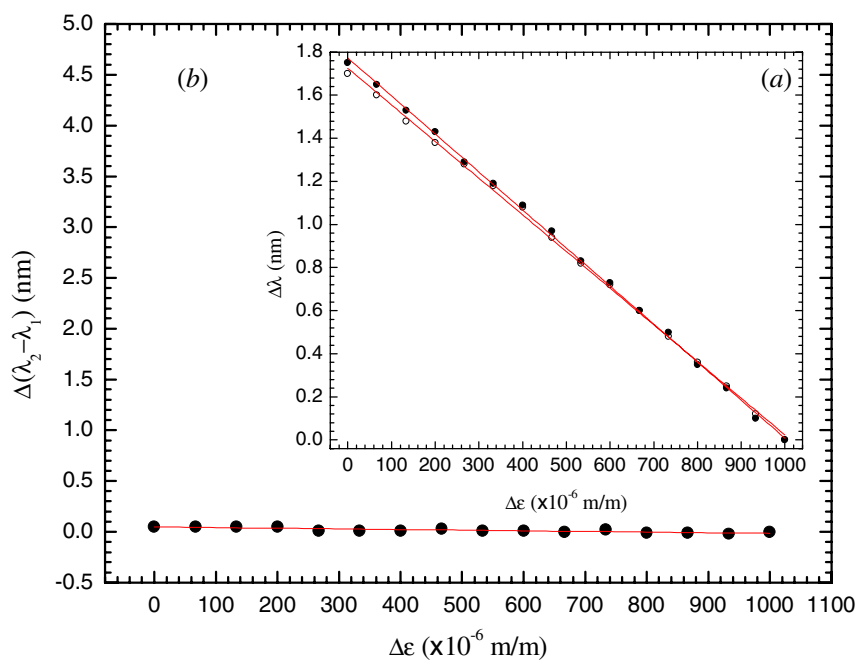

Figure 5. Strain response for $(a)$ the two loss bands centered at $\lambda_{1}$ and $\lambda_{2}$, and $(b)$ the measurement parameter, $\Delta\left(\lambda_{2}-\lambda_{1}\right)$.

embedded on a flexible cantilever beam, was developed as a temperature-insensitive directional bending sensor; the wavelength spacing between the multiple resonant peaks was not changed by the temperature applied, while a curvature sensitivity of $0.63 \mathrm{~nm} \mathrm{~m}$ could be attained [14]. In another approach, a pair of D-type fiber Bragg gratings (FBG), placed side by side along the plane defined by the D-shape cladding, were used for bending [15]; due to the fiber birefringence, each FBG presented two resonant peaks. Using the spectral separation between the two peaks, this geometry provided a differential sensitivity to curvature of about $0.07 \mathrm{~nm} \mathrm{~m}$, with insensitivity to temperature and axial strain. Improved curvature results can be attained by means of long period grating (LPG) structures. A temperature-insensitive LPG fabricated in an endlessly singlemode photonic crystal fiber proved to possess a bend sensitivity of $3.7 \mathrm{~nm} \mathrm{~m}$, along with no measurable change of the attenuation band with temperature [16]. Therefore, the singlemode-multimodesinglemode fiber structure described, under the proposed interrogation approach, can function as a highly sensitive curvature sensor, with residual cross-sensitivity to temperature and strain.

\section{Conclusions}

A temperature- and strain-independent curvature sensor, based on MMI, was presented in this communication. The proposed sensing device is based on an SMS fiber structure operating in transmission. The optical spectrum presents two narrow spectral loss bands-which shift with similar sensitivities to temperature and strain, but different sensitivities to curvature. The interrogation technique considered is based on definition of the measurement parameter as the wavelength difference of the two resonant loss bands; this parameter shows negligible sensitivity to temperature or strain $\left(0.3 \pm 0.1 \mathrm{pm}{ }^{\circ} \mathrm{C}^{-1}\right.$ and $(-0.06 \pm 0.01) \times 10^{-6} \mathrm{~m} \mathrm{~m}^{-1}$, respectively), but substantial sensitivity to curvature $(8.7 \pm 0.1 \mathrm{~nm} \mathrm{~m})$. This residual crosssensitivity to temperature and strain indicates that this sensing 
structure possesses great potential for curvature measurement in a wide range of applications.

\section{Acknowledgments}

This work was partially supported by project MICROPHYTE (ref. PTDC/EBB-EBI/102728/2008), funded by EU and the Portuguese State, and coordinated by author FXM, and project COST 299, funded by EU. Author SS received a $\mathrm{PhD}$ fellowship (ref. SFRH/BD/47799/2008), also funded by EU and the Portuguese State, and supervised by author FXM and co-supervised by author JLS.

\section{References}

[1] Soldano L B and Pennings E C M 1995 Optical multi-mode interference devices based on self-imaging: principles and applications J. Lightwave Technol. 13 615-27

[2] Rahman B M A, Somasiri N, Themistos C and Grattan K T V 2001 Design of optical polarization splitters in a single-section deeply etched MMI waveguide Appl. Phys. B 73 613-8

[3] Paiam M R and MacDonald R I 1997 Design of phased-array wavelength division multiplexers using multimode interference couplers Appl. Opt. 36 5097-108

[4] Mohammed W S, Smith P W E and Gu X 2006 All-fiber multimode interference bandpass filter Opt. Lett. 31 2547-9

[5] Wang Q and Farrell G 2006 All-fiber multimode-interference based refractometer sensor: proposal and design Opt. Lett. 31 317-9
[6] Wang Q and Farrell G 2006 Multimode fiber based edge filter for optical wavelength measurement application Microw. Opt. Technol. Lett. 48 900-2

[7] Donlagic D and Zavrsnik M 1997 Fiber-optic microbend sensor structure Opt. Lett. 22 837-9

[8] Zhang J, Zhang Y, Sun W and Yuan L 2009 Multiplexing multimode fiber and Fizeau etalon: a simultaneous measurement scheme of temperature and strain Meas. Sci. Technol. 20065206

[9] Frazão O, Viegas J, Caldas P, Santos J L, Araújo F M, Ferreira L A and Farahi F 2007 All-fiber Mach-Zehnder curvature sensor based on multimode interference combined with a long-period grating Opt. Lett. 32 3074-6

[10] Wu Q, Semenova Y, Wang P, Hatta A M and Farrell G 2011 Experimental demonstration of a simple displacement sensor based on a bent single-mode-multimodesingle-mode fiber structure Meas. Sci. Technol. 22025203

[11] Kumar A, Varshney R K, Antony C S and Sharma P 2003 Transmission characteristics of SMS fiber optic sensor structures Opt. Commun. 219 215-9

[12] Wang Q, Farrell G and Yan W 2008 Investigation on single-mode-multimode-single-mode fiber structure J. Lightwave Technol. 26 512-9

[13] Kawano K and Kitoh T 2001 Introduction to Optical Waveguide Analysis (New York: Wiley) chapter 5 , pp 165-230

[14] Han Y 2009 Directional bending sensor with temperature insensitivity using a sampled chirped fiber Bragg grating J. Appl. Phys. 105063103

[15] Araújo F M, Ferreira L A, Santos J L and Farahi F 2001 Temperature and strain insensitive bending measurements with D-type fibre Bragg gratings Meas. Sci. Technol. 12 829-33

[16] Dobb H, Kalli K and Webb D J 2004 Temperature-insensitive long period grating sensors in photonic crystal fibre Electron. Lett. 40 657-8 